\title{
Research on Security Control Strategy of Electronic Information Network Transmission
}

\author{
Ren Siying \\ Jilin Justice Officer Academy, Changchun, Jilin, 130062
}

Keywords: Electronic Information, Network Transmission, Security

\begin{abstract}
With the rapid development of Internet technology and computer technology, Internet users are haunted by the use of data. In this paper, the network transmission of electronic information is regarded as the research object, the electronic information network transmission system and its characteristics and safety are analyzed, and some electronic information network transmission security control strategies are proposed, aiming to provide reference for the safe transmission of electronic information network.
\end{abstract}

\section{Introduction}

Nowadays, computer network technology has penetrated into various fields, and electronic information technology has also developed rapidly. At the same time, there are many security problems in the electronic information engineering. The security of electronic information network transmission has attracted much attention from all walks of life. There are many security uncertainties in the transmission of electronic information network, which have a negative impact on the development of China's economic field and social stability [1-2]. There are many reasons for the safety problems of electronic information transmission, which requires relevant technicians to find out the problems with professional knowledge and take corresponding solutions to solve problems, so as to reduce the risk of electronic information network transmission.

\section{Electronic Information Network Transmission System}

The electronic information network transmission system is a comprehensive system of collection network, computer, communication, transmission, control, information assessment and so on. In the era of big data, the transmission system of electronic information and network plays an important role in social development, in fact, the basic guarantee for efficient operation of individual life and work as well as enterprises and units [3]. The electronic information network transmission system is an important basis for the development of the Internet and artificial intelligence, and the stability of the system is related to everyone's life and work.

\section{Transmission Characteristics of Electronic Information Network and Analysis of Its Security}

\subsection{Characteristics of electronic information network transmission}

Electronic information engineering is a popular field of current social development. It is a scientific and reasonable electronic processing of collected data. This technology also facilitates information collection, storage and management. The transmission of electronic information network has the characteristics of convenient, accurate, wide coverage and so on.

\subsubsection{Convenient}

In the transmission of electronic information network, data transmission is carried out by using specific hardware facilities and commands through the channel, so that it can load large quantities of data transmission work and make the Internet users more convenient to process and collect the data needed. Compared with the brain, the electronic information network transmission system can 
process huge data system, and its processing speed and efficiency are the most notable characteristics [4]. In addition, the continuous development of the material field makes the hardware and equipment conditions necessary for the electronic information transmission. Based on the development of electronic information network transmission, the transmission speed and efficiency have also been improved qualitatively, so users can use and get data more conveniently.

\subsubsection{Precision.}

Accuracy is another feature of electronic information network transmission. Electronic information engineering can set specific information processing results by analyzing and processing different information, so that it is convenient to check the transmission of electronic information and ensure the accuracy of its transmission. Compared with manual processing data, electronic information engineering can avoid the error caused by human factors and reduce the trouble of users because of inaccurate information [5].

\subsubsection{Wide coverage}

The transmission of electronic information network is widely used in all walks of life for data processing and data transmission. In the era of big data, our need for massive data has promoted the transmission of electronic information engineering and electronic information network. So the application of electronic information network transmission has been expanded.

\subsection{Transmission security of electronic information network}

The most prominent factor in the transmission of electronic information network is the hidden danger of network transmission lines and vulnerabilities in information system. Hackers are attacking the information system by some abnormal means after finding loopholes in the system. In 2017, hackers extended black hands to college students, threatening students to pay the corresponding ransom to retrieve the documents in the computer. The hacker event not only causes a certain loss to the users, but also makes the entire electronic information network transmission system unrest and brings unsafe hidden danger to the social harmony and stability. This requires that electronic information network transmission technicians should do everything possible to exclude all hidden dangers and ensure the safety of electronic information technology network transmission [6-7]. Once the security risks are found, it is necessary to use the means of firewalls to intercept the hidden dangers in the "outside of the wall" to ensure the security of the data transmission process. In the application of electronic information engineering, we need to protect the private network and the shared network, and make full use of computer technology to make the entire network data transmission system in a stable state, and then there is a certain security guarantee for the transmission of electronic information network.

\section{Security Control Strategy for Electronic Information Network Transmission}

\subsection{Technical integration aspect}

In the era of large data, the traditional computer system technology is obviously difficult to meet the needs of the development of the market economy and the transmission of information. This is because in traditional computer systems, the information transmission way of point to point letters cannot carry a lot of information, which is obviously inconsistent with the requirements of social development for information transmission. The important way to effectively solve the problem of the shortage of transmission information is to combine the traditional information transmission technology with the existing multicast information technology. First, we must understand the importance of electronic information system and its usage and information transmission control technology, and then clarify the importance of them in the electronic information network transmission system [8]. Master the information transmission principle of "point to point", utilize the advantages and advantages of point information transmission technology, and further analyze the information transmission technology of point based on this. Whether the information transmission technology of the research point can make up for its shortcomings in the multicast 
information transmission technology. Through in-depth and systematic research of point information transmission technology and multicast information transmission technology, the two technologies are effectively integrated, and then optimized and perfected information transmission control technology, which has important guarantee for the transmission safety of electronic information network.

\subsection{Transmission aspects of information transmission}

The development of electronic information network transmission requires that the operation of computer electronic information system should be fast and efficient. This is also a fundamental requirement for the development of information age. This requires that in the construction of electronic information network transmission system, we should not only optimize the information transmission technology, but also set up the transmission mode of information transmission reasonably. This is because in different signal frequencies, transmission rate difference of electronic information in the network transmission channel is more obvious, which resulted in the electronic transmission of information is difficult to grasp and practice to ensure that it has certain effects on the electronic transmission of information, and may make point security risks. Under the uncertain factors, the timeliness and stability of electronic information is unavoidable. Because of the consideration of the safety of transmission of electronic information network, we must take corresponding measures to avoid such a situation [9]. To ensure the timeliness and stability of information transmission, we must divide the information transmission channel and build a hierarchical channel system, so that information can be sent from high to low and sent to different levels of channels. In addition, an independent buffer program should be set up for each channel to ensure that information transmission has a certain buffer time, which ensures the stability and timely performance of electronic information in network transmission, and then ensures the safety of information transmission [10].

\subsection{Functional modules}

Functional modules play an important role in the transmission system of electronic information network. By its important status, it can be seen that it is also an important place for the safety control of electronic information network transmission. The functional modules mainly include: 1) channel optimization; 2) information release; 3) information state detection; 4) protocol decomposition and analysis; 5) information security processing. Taking the former electronic information transmission system as the research object, we can know that there is a coupling phenomenon among the functional modules in the past electronic information transmission system. In order to reduce the dependency between the functional modules, the corresponding measures must be taken to enable the functional modules to be applied to the system flexibly and effectively. In addition, in optimizing electronic information network transmission control technology, we need to improve the overall level of the electronic information network transmission system, and examine the needs of the system in application. Loosely coupled design is a method of optimizing the design of functional modules. In the application of loosely coupled design, we should focus on evading the dependencies of all functional modules, and have a clear location for each functional module, so as to ensure that each module can effectively play its functions and functions. Such electronic information transmission rate is guaranteed, of course, the electronic information network transmission security also has a thick protection umbrella.

\section{Conclusion}

Electronic information network transmission security of entire network environment harmony, which requires technical personnel should use professional skills to avoid unsafe factors in the construction of electronic information network transmission system, ensure the stability and security of information network transmission, promote harmonious development of electronic information network transmission. 


\section{Acknowledgements}

This paper would like to thank the teaching reform of education and adult education in the education department of Jilin province in 2017. Project approval number: 2017ZCY170.

\section{References}

[1] Pan S, Liu D, Zhu H, et al. Optimization control of active distribution network based on Photovoltaic forecast information[C]// China International Conference on Electricity Distribution. IEEE, 2014:594-597.

[2] Mouton A, Breckon T P. A review of automated image understanding within 3D baggage computed tomography security screening[J]. Journal of X-ray science and technology, 2015, 23(5):531-55.

[3] Kim H, Macredie J G R. A Qualitative Study of Stakeholders' Perspectives on the Social Network Service Environment[J]. International Journal of Human-Computer Interaction, 2014, 30(12):965-976.

[4] Kuzlu M, Pipattanasomporn M, Rahman S. Communication network requirements for major smart grid applications in HAN, NAN and WAN[J]. Computer Networks, 2014, 67(10):74-88.

[5] Zhang X, Qi F, Sun S, et al. Hybrid Multicast Transmission for Public Safety Network in 5G[J]. Mobile Information Systems, 2016, (2016-12-18), 2016.

[6] Naghavi S V, Safavi A A, Khooban M H, et al. A robust control strategy for a class of distributed network with transmission delays: LMI-based model predictive controller[J]. Compel-the International Journal for Computation \& Mathematics in Electrical \& Electronic Engineering, 2016, 35(5):1786-1813.

[7] Liu W X, Yu S Z, Tan G, et al. Information-centric networking with built-in network coding to achieve multisource transmission at network-layer[J]. Computer Networks, 2015, 115:110-128.

[8] Liu X, Li W, Zhao Z, et al. Tangential Network Transmission Theory of Reflective Metasurface with Obliquely Incident Plane Waves[J]. IEEE Transactions on Microwave Theory \& Techniques, 2017, PP (99):1-9.

[9] Liu Y N, Zhu M. Load-balance and coding awareness protocol multicast in space information network[C]// IEEE International Conference on Electronic Information and Communication Technology. IEEE, 2017:406-410.

[10] Reis Z S N, Gaspar J D S, Souza A C D, et al. Electronic Systems Interoperability Study: Based on the Interchange of Hospital Obstetrical Information[C]// IEEE, International Symposium on Computer-Based Medical Systems. IEEE, 2015:201-204.

\section{Author}

Ren Siying, August 23, 1983, female, han nationality, Changchun city, Jilin province, master's graduate, lecturer, research direction: application research of information system. Ji Lin Justice Officer Academy, unit address: Ji Lin Justice Officer Academy, no.1 Jingyuan road, Changchun economic development zone, Changchun city, Jilin province, post code: 130062.

Subject: Teaching reform of education and adult education in the education department of Jilin province in 2017. Project approval number: 2017ZCY170 